\title{
JAG1 loss-of-function mutations contributed to Alagille syndrome in two Chinese families
}

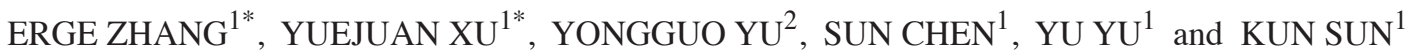 \\ Departments of ${ }^{1}$ Pediatric Cardiology and ${ }^{2}$ Pediatric Endocrinology and Genetics, Shanghai Institute for Pediatric Research, \\ Xinhua Hospital, School of Medicine, Shanghai Jiaotong University, Shanghai 200092, P.R. China
}

Received November 16, 2017; Accepted June 11, 2018

DOI: $10.3892 / \mathrm{mmr} .2018 .9217$

\begin{abstract}
Alagille syndrome (ALGS) is primarily caused by jaggedl (JAGl) mutations, $70 \%$ of which are protein-truncating mutations. However, no mutation hotspots have been discovered, and the pathogenic mechanism is not fully understood. The aim of the present study was to analyze two protein-truncating JAGl mutations detected in three Chinese ALGS patients. Mutation c.1261delT (p.Cys421Valfs) was identified in one patient with hepatic damage, xanthomas, facial abnormalities and cardiovascular defects, which was inherited from his father. The other mutation, c.1382_1383delAC (p.Asp461Glyfs), carried by a pair of monozygotic twins with hepatic damage, facial abnormalities and cardiovascular defects, was de novo. Biological experiments were performed to study the characteristics and function of these mutations. The p.Cys421Valfs and p.Asp461Glyfs mutant proteins appeared to be truncated in western blotting using anti-Flag bound to the N-terminus of JAG1. The RBP-JK-responsive reporter gene assay was used to investigate the ability of mutant JAG1 proteins to activate the Notch signaling pathway. The mutant proteins had a lower luciferase activity than the wild-type, indicating impaired transcriptional activation ability. Western blotting using soluble JAG1 from the culture medium revealed that the expression levels of the mutant proteins were lower than that of the wild-type, suggesting that less mutant JAG1 protein underwent proteolytic cleavage than the wild-type. In conclusion, these two loss-of-function JAG1 mutations may be associated with ALGS manifestations in these patients.
\end{abstract}

Correspondence to: Dr Kun Sun, Department of Pediatric Cardiology, Xinhua Hospital, School of Medicine, Shanghai Jiaotong University, 1665 Kongjiang Road, Shanghai 200092, P.R. China E-mail:sunkun@xinhuamed.com.cn

*Contributed equally

Key words: Alagille syndrome, jagged 1 gene, protein-truncating mutation, Notch signaling pathway

\section{Introduction}

Alagille syndrome (OMIM no. 118450), also known as arteriohepatic dysplasia, is a rare and complex autosomal dominant disorder characterized by developmental abnormalities of the heart, liver, vertebrae, eyes and face. The incidence of ALGS was estimated to be at least 1:70,000 live births $(1,2)$. Traditionally, the diagnosis of ALGS is based on finding of interlobular bile duct paucity in liver biopsy, accompanied by at least 3 of 5 major clinical features: Chronic cholestasis, congenital heart defects, ocular abnormalities (posterior embryotoxon), vertebral defects (butterfly vertebra) and characteristic facial phenotypes (pointed chin, broad forehead and bulbous nose) (3-5). Renal and vascular abnormalities are less commonly seen in a smaller percentage of cases (4). The phenotype of this disorder is highly variable, ranging from no apparent clinical involvement to severe disease leading to death. Therefore, the diagnosis of ALGS is impaired by its highly variable expressivity.

Two members of the Notch signaling pathway-JAG1 and NOTCH2-have been implicated in ALGS (6-8). The Notch signaling pathway is evolutionarily conserved, and is involved in both cell fate determination and organogenesis (9). The $J A G 1$ gene encodes the JAG1 protein belonging to the family of Notch ligands, and NOTCH2 encodes one of the four Notch receptors. Up to $94 \%$ of ALGS cases are caused by mutations of the JAGl gene (10). Only $1 \%$ cases are caused by NOTCH 2 mutations, in which renal malformations may be more common, but heart, skeletal and facial feature anomalies may be less frequent $(11,12)$. More than $400 \mathrm{JAGl}$ mutations have been identified to date, including whole gene deletions, splice site alterations, and missense, nonsense and frameshift mutations. Among JAG1 mutations, up to $70 \%$ have a premature termination codon (PTC), leading to a truncated JAG1 protein (13), or degradation by nonsense-mediated mRNA decay (NMD) (14). Loss-of-function mutations have been reported due to a truncated protein missing critical motifs (15).

Haploinsufficiency is considered to be a pathogenic mechanism of ALGS, since the same phenotypic features can be observed in patients with complete gene deletion, missense mutations, or protein-truncating mutations. Morrissette et al (16), reported that mutant JAG1 proteins R184J and L37S associated with functional haploinsufficiency, resulting from abnormal 
glycosylation patterns. Boyer-Di Ponio et al (15), showed that the missense mutant protein $\mathrm{C} 284 \mathrm{~F}$ as well as truncated proteins E1003X and $\Delta 4$ impaired activation of Notch signaling, suggesting that a dominant-negative effect mechanism may also explain the impaired biological function of some ALGS mutations. Biomarker detection was widely used to monitor the development of certain diseases (17-19). The finding that the developing heart was more sensitive to a decreased dosage of JAG1 than the developing liver implicates JAG1 as a plausible candidate biomarker for cardiac severity in ALGS (20). Further studies are needed to discover a more efficient and reliable detection method.

Here, we identified two protein-truncating mutations in two twins and one unrelated patient with ALGS. Expression level and transcriptional activation ability of these mutations were studied to illuminate their pathogenic mechanism in ALGS.

\section{Materials and methods}

Subjects. Eight individuals (three ALGS patients, their siblings and parents) from two families were enrolled for clinical and genetic studies (Fig. 1). All of them were of Han ethnicity. The project was approved by the Medical Ethics Committee of Xinhua Hospital (Shanghai, China). Written informed consents were provided by the participants or their legal guardians. Peripheral venous blood samples were collected after written informed consents were obtained. Control population was 100 healthy children undergoing routine physical examination, ethnically matched with patients. Genomic DNA was isolated from peripheral lymphocytes using a QIAamp DNA Blood Mini kit according to the manufacturer's instructions (Qiagen $\mathrm{GmbH}$, Hilden, Germany) and stored at minus $80^{\circ} \mathrm{C}$.

Whole-exome sequencing and analysis. SNPs tests showed that the two patients in family 2 were monozygotic twins, so genomic DNA sample of the proband (P3-IIB:3 in family 2) was subjected to whole-exome sequencing. The sample underwent whole-exome capture using the Sureselect Human All Exon kit (Agilent Technologies, Inc., Santa Clara, CA, USA), followed by sequencing on the Illummina HiSeq 2500 sequencer (Illumina, Inc., San Diego, CA, USA) with an $\sim 100 x$ depth of coverage. Sequence data were aligned and mapped to the human genome reference (hg19). Variants (the single nucleotide variants and insertions/deletions) were called by SAMTOOLS (samtools.sourceforge.net/) and compared with common variant database, including dbSNP135 (ncbi.nlm.nih.gov/) and 1000 genomes project database (www.1000genomes.org/).

Mutation validation by Polymerase Chain Reaction (PCR) and Sanger sequencing. PCR and Sanger sequencing was used to detect possible variants in family 1 and to confirm the candidate variant in family 2. Parents or siblings were screened for the specific variants in the affected exons by sequence analysis. DNA from 100 healthy controls were also screened. Primers that amplify 26 exons (RefSeq NM_000214.2) and at least 100 bp of intron-exon boundaries were designed (primer sequences available on request) by Primer Premier v.5.0 (Premier Biosoft International, Palo Alto, CA, USA). Standard PCR were performed with $100 \mathrm{ng}$ genomic DNA using MyTaq ${ }^{\mathrm{TM}}$ HS DNA Polymerase (Bioline Reagents Ltd., London, UK) on an Applied Biosystems Veriti instrument (Applied Biosystems, California, USA). PCR products were sequenced on an Applied Biosystems 3730 sequencer (Applied Biosystems; Thermo Fisher Scientific, Inc.) and sequence traces were compared with the reference sequence using the GenBank BLAST program (blast.ncbi. nlm.nih.gov/Blast.cgi). Mutation nomenclature is based on the transcript NM_000214.2 and NP_000205.1.

Plasmid construction. pCMV3-Flag-JAG1 vector containing human JAG1 cDNA was bought from Sino biological inc as a template, the FLAG tag was at the N-terminus of the open reading frame (ORF) and the signal peptide was at the $\mathrm{N}$-terminus of the FLAG tag. The c.1261delT (p.Cys421Valfs), c.1382_1383delAC (p.Asp461Glyfs) mutant expression vectors were made using Quickchange Site-Directed Mutagenesis kit (Agilent Technologies, Inc.). Complementary primers were synthesized containing the desired mutation flanked by unmodified sequences for the mutagenesis reaction. Forward primers for these reactions were as follows, p.Cys421Valfs: 5'-GAATGTGAGGCCAAACCTGTGTAAACGCCAAAT CC-3'; p.Asp461Glyfs: 5'-CAGTGTCAGAATGGCCTCCTG TCGG-3'. All plasmids used for transfection were determined by NanoDrop 2000 Microvolume Spectrophotometers (Thermo Fisher Scientific, Inc.), and according to our previous studies $(21,22)$, the concentration was controlled at $500 \mathrm{ng} / \mu 1$.

Cell culture and transfection. NIH-3T3 cells (3,4,20) (Cell Bank of the Chinese Academy of Science, Shanghai, China) were cultured in Dulbecco's modified Eagle's Medium (HyClone; GE Healthcare Life Sciences, Logan, UT, USA) supplemented with $10 \%$ fetal bovine serum (MP Biomedicals, Santa Ana, California, USA), penicillin (100 units/ml) and streptomycin $(100 \mu \mathrm{g} / \mathrm{ml})$ at $37^{\circ} \mathrm{C}$ in $5 \% \mathrm{CO}_{2}$. Cells were transfected using Fugene HD Transfection Reagent (Promega Corporation, Madison, WI, USA) according to the manufacturer's instructions.

Reverse transcription-quantitative PCR (RT-qPCR). Cells were seeded in 12-well plate for $24 \mathrm{~h}$, then transfected with $1.1 \mu \mathrm{g}$ pCMV3-C-FLAG vector, or with vector expressing either wild-type $J A G 1$ or $J A G 1$ mutants (p.Cys421Valfs, p.Asp461Glyfs). After $36 \mathrm{~h}$, total RNA was extracted by RNAiso (Takara Bio Inc., Otsu, Japan) following manufacturer's instructions, and $1 \mu \mathrm{g}$ of that RNA was reverse transcribed into cDNA using Prime Script RT Master Mix (Takara Bio Inc.). The mRNA level of JAGl was quantified by RT-qPCR using SYBR Premix Ex Taq (Takara Bio Inc.) on an Applied Biosystems 7500 system. Reaction volume was $20 \mu \mathrm{l}$ containing $100 \mathrm{ng}$ reverse transcript product, $0.2 \mu \mathrm{M}$ primers (spanning exon-exon junction), $10 \mu \mathrm{l} \mathrm{SYBR}$ Premix Ex Taq and $0.4 \mu 1$ ROX Reference DyeII. PCR consisted of an denaturation step at $95^{\circ} \mathrm{C}$ for $30 \mathrm{sec}$, followed by 40 cycles amplification step $\left(95^{\circ} \mathrm{C}\right.$ for $5 \mathrm{sec}, 60^{\circ} \mathrm{C}$ for $\left.34 \mathrm{sec}\right)$. The relative quantitation of JAG1 mRNA was determined using $2^{-\Delta \Delta \mathrm{Cq}}$ method (23) and normalized by an internal control GAPDH. Primers amplifying cDNA of $J A G 1$ and $G A P D H$ were as follows, JAG1-F: 5'-CAACCGCATCGTGCTGC-3', JAG1-R: 5'-CGCCTCCACAAGCAACGTAT-3'; GAPDH-F: 5'-AGG 
A

IA

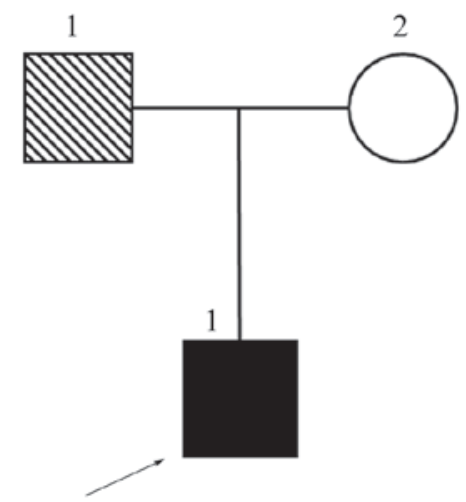

$\mathrm{C}$

$\begin{array}{ccc} & \text { Sex/Age } & \text { Liver } \\ & & \\ \text { P1-IIA:1 } & \text { M/5 } & + \\ & & \\ \text { P2-IIB:2 } & \text { F/2 } & + \\ & & + \\ & & + \\ \text { P3-IIB:3 } & \text { F/2 } & +\end{array}$

B

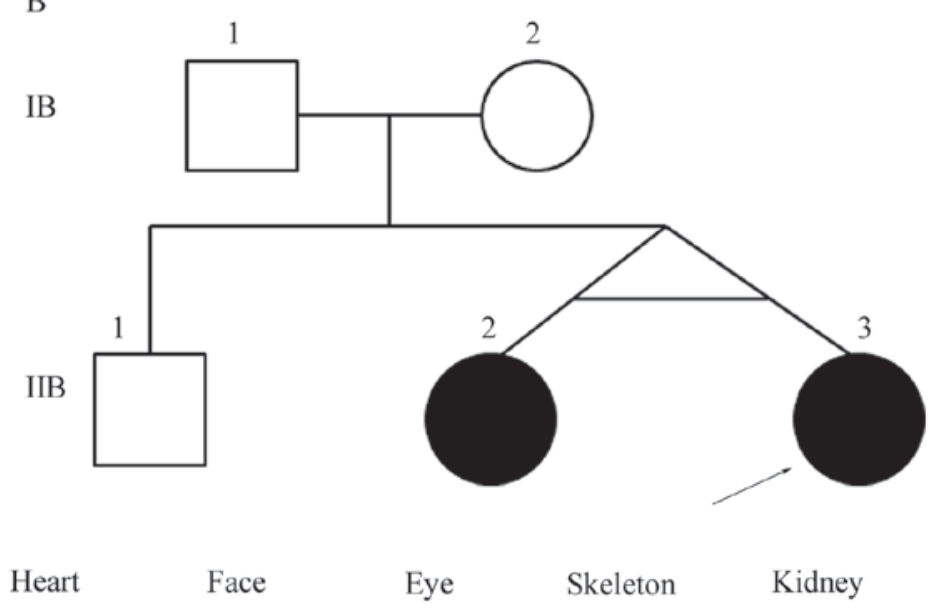

$+$

$+$

$+$

Figure 1. Pedigrees of the two ALGS families and symptoms of the three patients. Pedigrees of (A) family 1 and (B) family 2, respectively. (C) The symptoms of the three patients. The hatched symbol indicates the father with mild liver damage. I and II refer to the first and second generations of the families, respectively. Arrows indicate the probands. Squares indicate males and circles indicate females. White symbols are unaffected individuals, and black symbols represent the patients. ALGS, alagille syndrome.

\section{TCGGTGTGAACGGATTTG-3', GAPDH-R: 5'-TGTAGA CCATGTAGTTGAGGTCA-3'.}

Western blot analysis. Cells were grown in 12-well plates and transfected with $1.1 \mu \mathrm{g}$ empty vector, or with vector expressing either wild-type JAG1 or mutant proteins. For protein from cell lysates, cells were harvested $48 \mathrm{~h}$ later, lysed by RIPA lysis buffer (Beyotime Institute of Biotechnology, Shanghai, China) and PMSF (Beyotime Institute of Biotechnology). Supernatant was collected after centrifugation (16,000 g, 20 min). Protein concentration was determined using Enhanced BCA Protein Assay kit (Beyotime Institute of Biotechnology). Then $20 \mu \mathrm{g}$ of protein was loaded onto a 7.5\% SDS-polyacrylamide gel and subsequently transferred onto a nitrocellulose membrane (EMD Millipore, Billerica, MA, USA), blocked and incubated with rabbit anti-FLAG (1:1,000; Sigma-Aldrich; Merck KGaA) or anti-GAPDH (1:10,000; Sigma-Aldrich; Merck KGaA) overnight at $4^{\circ} \mathrm{C}$. After washing three times in TBS/Tween 20, the membrane was incubated with horseradish peroxidase conjugated anti-rabbit secondary antibody (1:10,000; Jackson ImmunoResearch Laboratories, Inc., West Grove, PA, USA) for $1 \mathrm{~h}$ at room temperature. Proteins were revealed by Immobilon Western Chemiluminescent HRP Substrate (EMD Millipore). Densitometry of the bands was performed by Image Lab (Bio-Rad Laboratories, Inc., Hercules, CA, USA). The relative intensity of the wild-type band was set to 1 as a standard reference. Based on this, relative intensities \pm SEM were calculated for the two mutant protein bands.
Luciferase assays. RBP-J $\mathrm{K}$ is an important transcriptional factor in the Notch signaling pathway. When the JAG1 protein interacts with the Notch receptor, the Notch intracellular domain (NICD) releases and combines with RBP-Jא, shifting RBP-JK from a transcriptional repression state to a transcriptional activation state. Thus, we used a RBP-Jא-responsive reporter gene assay (SABiosciences; Qiagen $\mathrm{GmbH}$ ) to investigate the ability of mutant JAG1 proteins to activate the Notch signaling pathway.

After being cultured in 48-well plates for $24 \mathrm{~h}$, cells transfected with pCMV3 control vector or with vector expressing JAG1 (wild-type or mutant proteins) were used as 'signal sending cells', while cells transfected with pMyc-C-CMV5-NOTCH3 plasmid (kindly provided by Dr. M. Tada, National Institute of Health Sciences, Japan) and RBP-JK-responsive luciferase reporter plasmid were used as 'signal receiving cells'. The following day, cells were harvested by trypsin-EDTA and counted, then signal sending cells and signal receiving cells were co-cultured in a 24 -well plates at the ratio of 1:1 for another $24 \mathrm{~h}$ as described previously (3). Luciferase activities were measured using a Dual-Glo luciferase assay system (Promega Corporation, Madison, WI, USA) according to the manufacturer's instructions. Firefly luciferase activity levels were normalized to that of Renilla. All assays were repeated three times.

Extraction of protein from culture medium. For protein from culture medium, $6 \mathrm{~h}$ after transfection, the medium was 
A

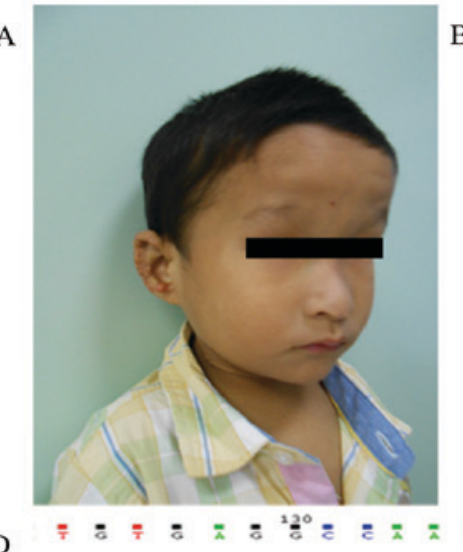

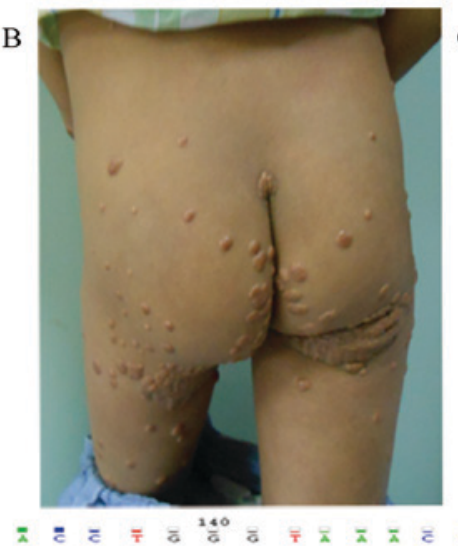

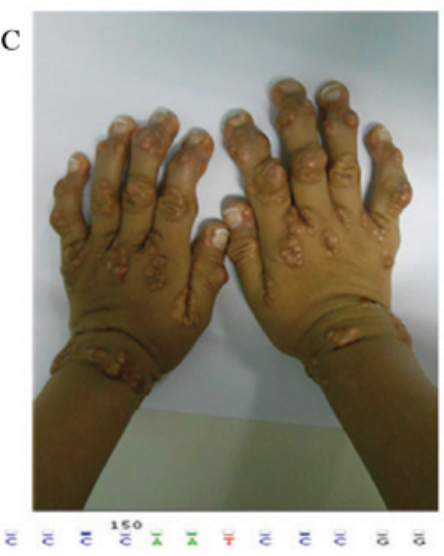

a

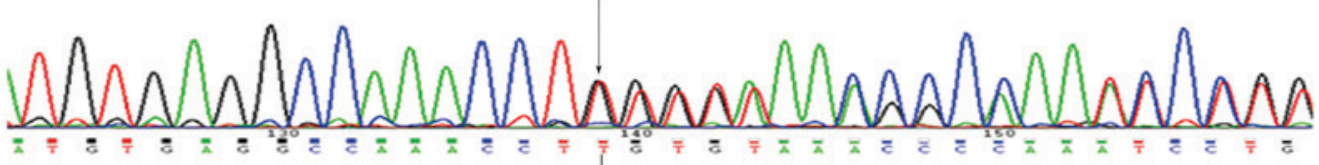

b

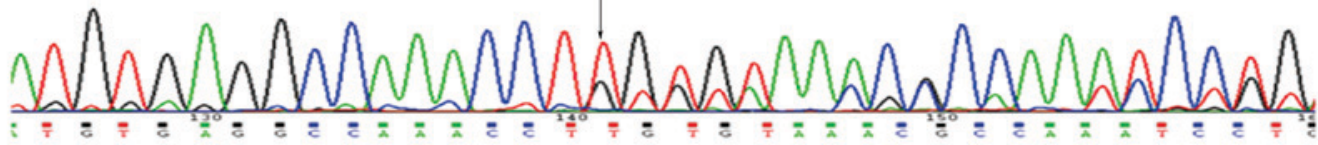

c

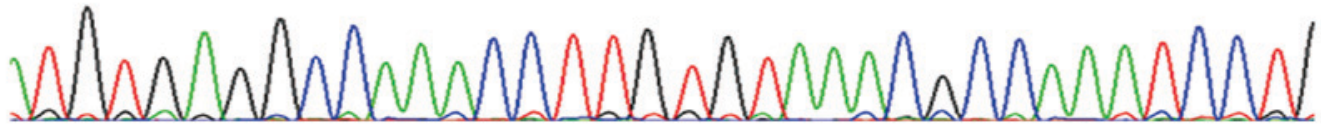

Figure 2. Phenotype and DNA sequencing results of family 1. (A) The facial abnormalities of Patient 1-IIA:1 included a prominent forehead, deep-set eyes, a bulbous nose and a pointed chin. Severe xanthomas were also observed on the (B) buttocks and (C) hands. (D) The c.1261delT mutation in family 1 ; a-c represent the DNA sequences of the proband, his father and his mother, respectively. Arrows indicate the position of the base deletion.

replaced with serum-free medium and cultured for $48 \mathrm{~h}$. The medium was collected in an Amicon Ultra-4 centrifugal filter (50 kDa for wild-type and $10 \mathrm{kDa}$ for 421fs and 461fs; EMD Millipore) and centrifuged at 3,500 g for $40 \mathrm{~min}$. The filtrate was collected to study JAG1 expression levels in supernatant by western blotting. Densitometry of the bands was performed as described above.

Statistical analysis. Data are presented as the mean \pm standard error of the mean of three independent experiments. Statistical differences between groups were analyzed by one-way analysis of variance with Tukey's post hoc test for multiple comparisons using GraphPad Prism v.6.02 (GraphPad Software, Inc., La Jolla, CA, USA). P $<0.05$ was considered to indicate a statistically significant difference.

\section{Results}

Clinical manifestation. We performed clinical examinations on the two probands and their parents and siblings (Fig. 1). In family 1 , the proband (P1-IIA:1) was a five-year-old boy who had severe xanthomas (Fig. 2B and C). Hepatic cell damage and cholestasis were indicated by elevated biochemical indexes (Table I). He had facial abnormalities of a prominent forehead, deep-set eyes, a bulbous nose and a pointed chin (Fig. 2A). Echocardiography showed pulmonary branch stenosis and patent foramen ovale. X-ray examination of the spine did not reveal butterfly vertebrae or any other development anomalies in the thoracic or lumbosacral segments.
Ophthalmologic examination did not reveal posterior embryotoxon. His parents were apparently normal and not consanguineous. However serum biochemical tests of the father illustrated mild hepatic cell damage with slightly elevated serum levels of ALT 126, AST 48 and $\gamma$-GT 98 U/l.

In family 2, the proband (P3-IIB:3) is one of monozygotic twins. At 2 months of age, she had been referred to our hospital for persistent jaundice. Echocardiography showed supravalvular aortic stenosis and left pulmonary artery stenosis. She had a dysmorphic face, with a broad forehead, a bulbous nose and a pointed chin (Fig. 3A), but no skeleton or ocular malformations. Biochemical tests revealed elevated serum liver enzyme levels, and slightly elevated serum bilirubin and lipid levels (Table I). Her twin sister had face abnormalities, mild pulmonary branch stenosis and hepatic damage. The other family members had none of these features.

Whole-exome sequencing and analysis. Whole-exome sequencing of P3-IIB:3 in family 2 was at a mean depth of $\sim 100 x$, covering more than $95 \%$ of exome regions. After filtering the total 46497 single nucleotide variants (SNVs) and 3774 insertions/deletions (Indels), we located a frameshift deletion on exon 11 in JAG1 (c.1382_1383delAC, p.Asp461Gly). This mutation is presumed to result in a premature stop codon.

Direct Sanger sequencing. To validate the WES results from proband P3-IIB:3 and to detect mutations in proband P1-IIA:1, we performed PCR and Sanger sequencing on the patients and extended family members. Sanger sequencing confirmed the 
Table I. Clinical features of the probands.

Clinical indexes

Proband (P1-IIA:1)

Proband (P3-IIB:3)

Total bile acid (1-10 $\mu \mathrm{mol} / \mathrm{l})$

$\begin{array}{cc}284.3 & 313.0 \\ 140 & 60 \\ 139 & 51 \\ 829 & 814 \\ 829 & 310 \\ 132.1 & 27.6 \\ 59.5 & 18.4 \\ 21.53 & 8.73 \\ 5.40 & 2.59 \\ 7.14 & 4.01\end{array}$

ALT (0-75 U/l)

60

AST (8-38 U/l)

Alkaline phosphatase (34-114 U/l)

814

$\gamma$-GT (16-73 U/l)

310

Total bilirubin $(3.42-20.52 \mu \mathrm{mol} / \mathrm{l})$

27.6

Conjugated bilirubin $(0-6.8 \mu \mathrm{mol} / \mathrm{l})$

8.4

Total cholesterol (3.36-6.46 mmol/l)

2.59

$\operatorname{LDL}(2.07-3.1 \mathrm{mmol} / \mathrm{l})$

4.0

ALT, alanine aminotransferase; AST, aspartate aminotransferase; $\gamma$-GT, $\gamma$-glutamyl transpeptidase; LDL, low density lipoprotein cholesterol.
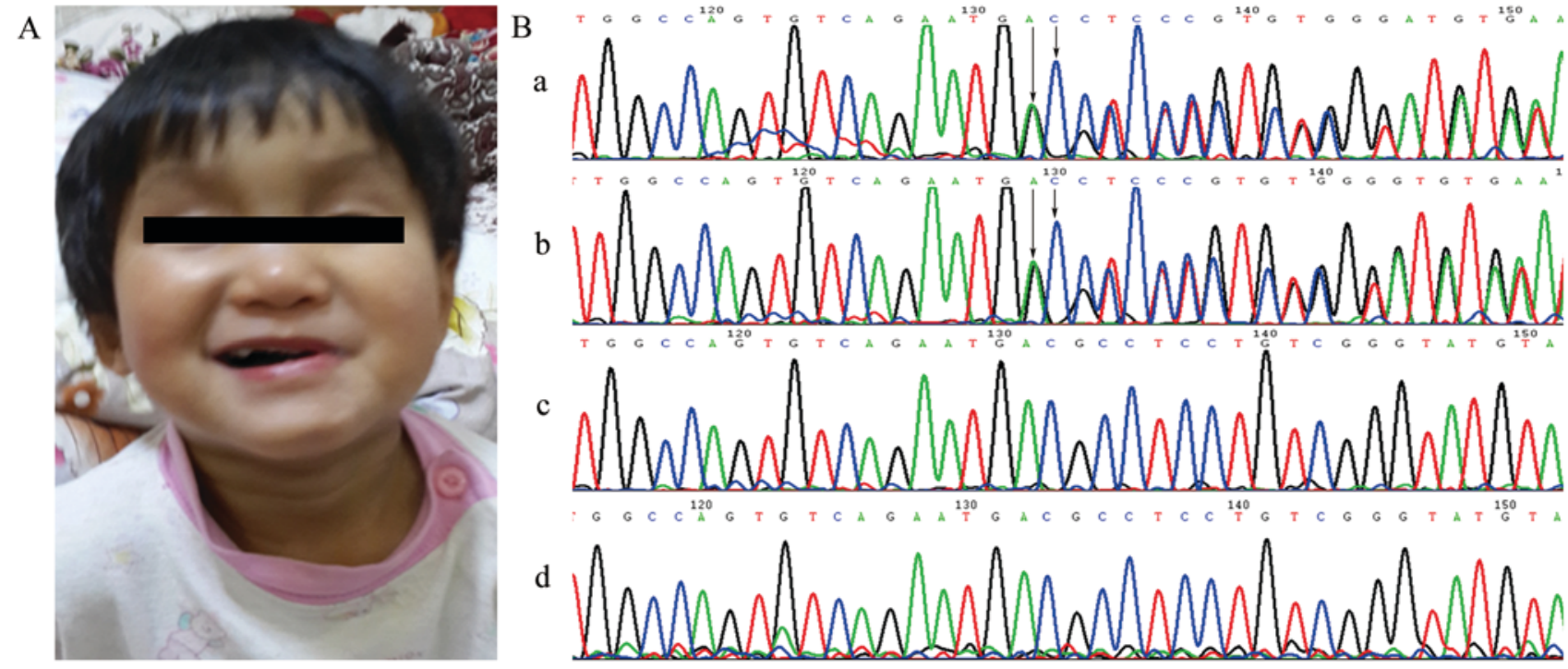

Figure 3. Phenotype and DNA sequencing results of family 2. (A) Patient 3-IIB:3 had facial anomalies including a broad forehead, a bulbous nose and a pointed chin. (B) The c.1382_1383 delAC mutation in family 2; a-d indicate the DNA sequences of the proband, her twin sister, her mother and her father, respectively. Arrows indicate the position of the base deletion.

two mutations: c.1261delT and c.1382_1383delAC in exons 10 and 11 of $J A G 1$, respectively (Figs. 2D and 3B). The c.1261delT (p.Cys421Valfs) frameshift mutation of JAG1 found in patient P1-IIA:1 (Fig. 2Da) was inherited from his father IA:1 (Fig. 2Db) who had mild hepatic cell damage, while his mother did not carry this mutation (Fig. 2Dc). The other frame-shift mutation c.1382_1383delAC (p.Asp461Glyfs), found in P2-IIB:2 (Fig. 3Bb) and P3-IIB:3 (Fig. 3Ba) was de novo, for it was not detected in their mother (Fig. 3Bc) or father (Fig. 3Bd). None of these mutations were detected in the 100 healthy controls. Mutation c.1261delT has not been reported in the Human Gene Mutation Database (www.hgmd.cf.ac.uk/) or the NHLBI Exome Sequencing Project (evs.gs.washington.edu/). While c.1382_1383delAC had been detected in a retrospective study (24), it had not been analyzed yet. Mutations c.1261delT and c.1382_1383delAC resulted in the conversion of the respective amino acid resides 421 , a cysteine and 461, an aspartate to stop codons, leading to the loss of subsequent domains.
Expression of JAG1 mutants. Relative quantitation of mRNA in control vector, wild-type and two mutants was analyzed using RT-qPCR (Fig. 4A). Expression levels of p.Cys421Valfs and p.Asp461Glyfs did not reveal any statistical difference with that of wild-type $(\mathrm{P}>0.05)$, indicating undisturbed mRNA transcription. We then performed western blotting using expression vector with a Flag tag at the N-terminus of JAG1 cDNA, thus truncated protein could be detected. As expected, mutants p.Cys421Valfs and p.Asp461Glyfs both appeared as truncated proteins (Fig. 4B, left panel): p.Cys421Valfs was $\sim 55 \mathrm{kDa}$ and p.Asp461Glyfs was $\sim 60 \mathrm{kDa}$, almost $130 \mathrm{kDa}$ smaller than the wild-type $(\sim 180 \mathrm{kDa})$. Densitometry revealed that relative intensity of the wild-type was lower than that of p.Cys421Valfs $(\mathrm{P}<0.001)$, but had no difference with that of p.Asp461Glyfs (Fig. 4B, right panel).

Transcriptional activation ability of JAG1 mutants. The RBP-JK-mediated the Notch signaling pathway transcriptional 

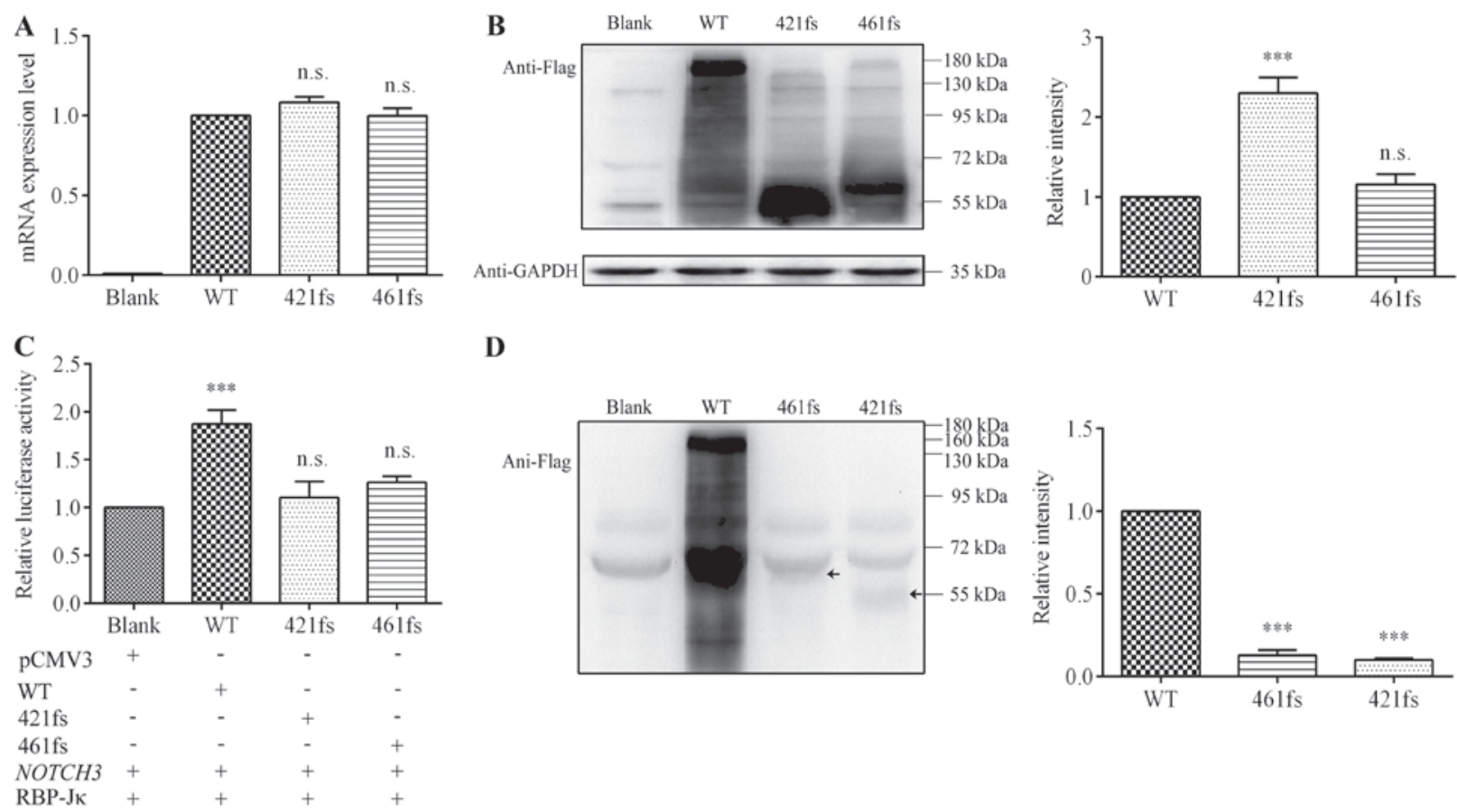

D
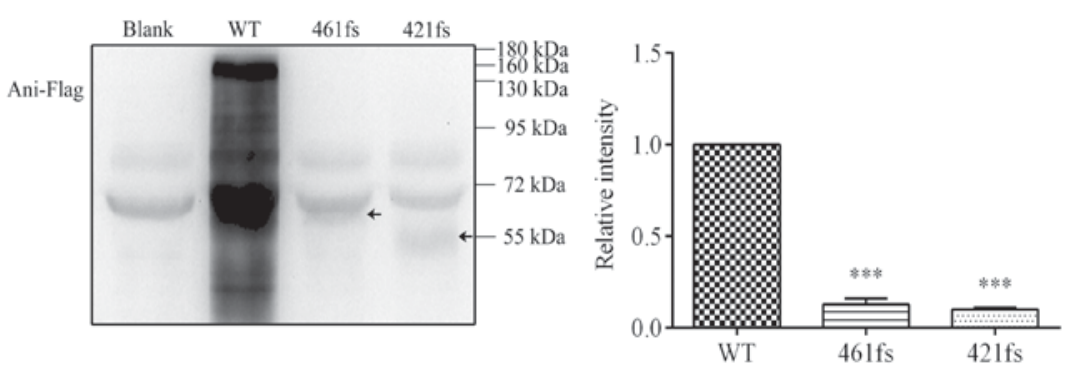

Figure 4. Analysis of expression characteristics and transcriptional activation ability of the mutations. (A) NIH-3T3 cells transiently transfected with pCMV3 vector (blank), WT JAG1 (WT group), p.Cys421Valfs (421fs group) and p.Asp461Glyfs (461fs group). Following $36 \mathrm{~h}$, RNA was isolated and reverse transcribed into cDNA. The mRNA expression levels of both 421fs and 461fs exhibited no difference with that of WT. (B) Flag-tagged WT, 421fs and 461fs mutant proteins were probed by anti-Flag immunoglobulin G by western blotting. Left panel reveals that the molecular weight of WT was $\sim 180 \mathrm{kDa}$, while $421 \mathrm{fs}$ $(\sim 55 \mathrm{kDa})$ and $461 \mathrm{fs}(\sim 60 \mathrm{kDa})$ were $\sim 130 \mathrm{kDa}$ smaller than WT, indicating impaired protein integrity. The right panel presents densitometry analysis of the bands; WT had lower relative intensity than $421 \mathrm{fs}$, though there was no difference with $461 \mathrm{fs}$. (C) When compared with the control group (blank), WT had a statistically significant increase in luciferase activity, suggesting activation of Notch signaling. Conversely, the luciferase activity of $421 \mathrm{fs}$ and $461 \mathrm{fs}$ was no different to that of blank, indicating impaired transcriptional activation ability. (D) WT and mutant JAG1 proteins were collected from the culture medium and analyzed by western blotting. WT appeared at $\sim 160 \mathrm{kDa}$, while $461 \mathrm{fs}$ and $421 \mathrm{fs}$ appeared at $\sim 60$ and $\sim 55 \mathrm{kDa}$, indicated by arrows, respectively (left panel), Relative intensities were calculated by comparing the intensity of each band with that of the WT (right panel). Data are presented as the mean \pm standard error of the mean. ${ }^{* * *} \mathrm{P}<0.001$ vs. WT. n.s., not significant; WT, wild type; JAG1, jagged 1.

activation was measured using a RBP-Jк-responsive luciferase reporter plasmid (Fig. 4C) 66. Wild-type JAG1 had a statistically significant increase (almost two-fold, $\mathrm{P}<0.001$ ) in luciferase activity compared to the control vector, suggesting over-expression of wild-type JAG1 protein successfully activated the Notch signaling pathway. Both p.Cys421Valfs and p.Asp461Glyfs had a lower activity than the wild-type, nearly the same as the control vector $(\mathrm{P}>0.05)$, indicating impaired transcriptional activation ability.

Protein expression level of JAG1 mutants in culture medium. After being transported to the plasma membrane, the JAG1 protein undergoes proteolytic cleavage, and the soluble extracellular fragment is secreted into the culture medium. In western blotting analysis, the wild-type appeared at $\sim 160 \mathrm{kDa}$, while p.Cys421Valfs and p.Asp461Glyfs appeared at $\sim 55 \mathrm{kDa}$ and $\sim 60 \mathrm{kDa}$, indicated by arrows, respectively (Fig. 4D, left panel). The relative intensities of both p.Cys421Valfs and p.Asp461Glyfs were much lower than that of the wild-type (Fig. 4D, right panel), suggesting less mutant JAG1 protein underwent proteolytic cleavage, thus fewer soluble fragments were secreted into the culture medium.

\section{Discussion}

Variable anomalies add intricacy to the differential diagnosis of ALGS, while advances in molecular genetics contribute to early diagnosis of the disease and improvement of survival rates. Protein-truncating mutations account for up to $70 \%$ of JAG1 mutations in ALGS patients. Nevertheless, no mutation hotspots have been identified yet $(13,24)$, and the pathological mechanisms of these kinds of mutations have been controversial. This study was aimed at analyzing the biological functions of two JAGl protein-truncating mutations detected in Chinese ALGS patients, which may allow for the possibility of future genetic counseling.

JAG1 is a single-transmembrane protein composed of five main structures, an intracellular region, a transmembrane domain and an extracellular section, which includes a signal peptide, an N-terminal region, and a DSL domain, followed by 16 epidermal growth factor-like (EGF-like) repeats and a $\mathrm{CR}$ region (25). The signal peptide associates with correct target of the JAG1 protein in the cell membrane, and the DSL domain is necessary in binding JAG1 to the Notch receptors (26). EGF-like repeats are evolutionarily highly conserved and have a vital role in maintaining conformation and stability of the protein, as well as facilitating the interaction between the ligand and the receptor (25). Mutations p.Cys421Valfs and p.Asp461Glyfs were located in the sixth and seventh EGF-like repeats, respectively. These frameshift deletions resulted in the conversion of the respective amino acid resides 421 , a cysteine and 461 , an aspartate to stop codons, leading to the loss of subsequent domains. The loss of the intracellular domain may affect adhesion between cells, 
inhibiting cell motility (26). Even when the signal peptide and the DSL domain remained intact, assays found that Serrate DSL mutants retained the ability to bind to the Notch receptor despite the loss of transactivation function, suggesting that binding alone does not ensure activation of the Notch signaling pathway (27). Therefore, it is highly suspected that these two mutations are the cause of the nonfunctional JAG1 protein.

The mRNA expression levels of p.Cys421Valfs and p.Asp461Glyfs were similar to that of the wild-type, suggesting that these mutations had no effect on normal transcription processes. Mutations with PTC are typical targets of NMD, which prevents deleterious proteins from being produced $(28,29)$. However, in some cases mRNAs may escape NMD for one or more rounds of translation, because of the inefficient recruitment of the NMD-activating complex $(30,31)$. Besides, Researches showed that in mammalian cells, the induction of NMD requires a long $3^{\prime}$ UTR or the presence of an exon-junction complex downstream of a PTC $(32,33)$. Thus, PTC in eukaryotic over-expression plasmid with intronless $J A G 1$ CDS will not subjected to NMD. To assess NMD, it is more convincing to test mRNA levels using patient-derived lymphoblasts and/or tissues (14), but unfortunately, due to social reasons, we could not obtain enough blood samples or tissues, so we studied the mRNA expression level of mutant JAG1 in transient transfected cell as previous researches did (34). We used western blotting to affirm the abnormality of the mutant JAG1 protein by detecting the Flag tag that was attached to the N-terminus of the JAGl ORF. Since thep. Cys421Valfs and p.Asp461Glyfs mutations express truncated JAG1 proteins, both of them had smaller molecular weights than the wild-type. Mutant protein p.Cys421Valfs had higher expression level than the wild-type, possibly due to posttranslational modification $(16,20)$.

The ability of JAG1 mutants to activate Notch signaling was measured by the reporter gene assay. The truncated mutants had lower luciferase activity than the wild-type, almost at the same level as the control vector, demonstrating a loss of function in the transcriptional activation process. After being transported to the plasma membrane, JAG1 can interact with the NOTCH receptor and initiate downstream transcription. Membrane-bound JAG1 protein undergoes proteolytic cleavage, secreting a soluble extracellular fragment into the culture medium. If the protein was not transported correctly to the plasma membrane, proteolytic cleavage might not occur, and the soluble extracellular fragment might not be secreted into the culture medium. Western blotting using wild-type and mutant JAG1 proteins from cell culture medium suggested that fewer soluble extracellular fragments were generated by either mutant protein than by the wild-type. To detect a possible cleavage process of the JAG1 protein, an anti-Jag1 antibody directly against the C-terminus should be used to identify the membrane associated and intracellular fragments of JAG1 (15). However, both p.Cys421Valfs and p.Asp461Glyfs were truncated without the transmembrane and intracellular domains, so it is difficult to analyze their proteolytic cleavage process directly. Tada et al (3) found that P163L and R184H localized in the endoplasmic reticulum, so their soluble forms were not detected in the culture medium, which resulted in a failure to activate RBP-Jк. Additionaly, trucated JAG1 protein E1003 exhibited decreased soluble protein levels with damaged transcriptional activity (15). This evidence suggests that abnormal localization of JAG1 may affect its regulation of down-stream target elements. Further studies of p.Cys421Valfs and p.Asp461Glyfs are still needed to elucidate the association between their subcellular localization and functional abnormality.

Lack or absence of Notch signaling affects development of those organs mainly involved in ALGS, such as liver, heart and face. A study by Sparks et al (35) found a dose-dependent relationship between impaired Notch signaling and diminished peripheral intrahepatic bile ducts (IHBD) derived from bipotential hepatoblast progenitor cells, suggesting that Notch signaling is a critical requirement for the formation of IHBD. Notch signaling is also closely implicated in cardiogenesis, as Notch mutant mouse models demonstrate various cardiac abnormalities, from septal defects to heart looping defects (36). Mice with attenuated JAG1 had midfacial hypoplasia phenotypes similar to ALGS, unveiling the necessity of Notch signaling in midfacial cranial neural crest cells during development (37). Consequently, we believe that the frameshift mutations are deleterious and might contribute to ALGS phenotypes.

This study is the first to analyze the biological function of two protein-truncating JAG1 mutations detected in Chinese ALGS patients. Similarities among the three ALGS patients are frameshift mutations in the EGF domain lead to truncated protein with function loss that are associated with manifestations involving liver and heart. There are also some differences. In family 1, follow-up with P1-IIA:1 after treatment with UDCA (ursodeoxycholic acid) indicated decreased bilirubin levels, but hepatic enzymes elevated significantly, and eventually the patient received a liver transplantation. His liver function has improved, and his xanthomas reduced. His father carried the same mutation, but had only sub-clinical findings: Slightly elevated liver enzymes that could result from profound reasons apart from ALGS. A cohort study showed that the frequency of serious clinical findings in relatives is much lower compared to the probands in their families (38). It still remains to be explored why the same mutation can lead to variable manifestations, from full exhibition of ALGS features to no detectable findings at all. In family 2 , the monozygotic twins carried a de novo mutation. This is a primary report of Chinese monozygotic twins with ALGS. The twins had similar face abnormalities, pulmonary branch stenosis and hepatic damage, but they did not finish their follow up, so we were unable to evaluate their present conditions. Nevertheless, future genetic counseling of the two families will still benefit from these findings.

This is the first study to analyze the biological function of two protein-truncating JAGl mutations detected in three Chinese ALGS patients. According to the results, mutations c.1261delT and c.1382_1383delAC produced truncated JAG1 proteins with impaired function in activating the Notch signaling pathway. These findings suggested that the two mutations might associate with ALGS manifestations in these patients, enriched the spectrum of JAGl mutation known in ALGS patients and provided necessary information for genetic counseling of families with ALGS patients. 


\section{Acknowledgements}

The authors would like to thank Dr. Minoru Tada (Division of Biological Chemistry and Biologicals, National Institute of Health Sciences, Tokyo, Japan) for generously offering the plasmids used in the present study.

\section{Funding}

The present study was supported by grants from the National Basic Research Program of China (grant no. 2010CB529501), the National Natural Science Foundation of China (grant nos. 81300068/H0201 and 81270233/H0204) and the Major Key Project for Fundamental Research from Shanghai Science and Technology Committee (grant no. 13JC1401705).

\section{Availability of data and materials}

The datasets used and/or analyzed during the current study are available from the corresponding author on reasonable request.

\section{Authors' contributions}

EZ and YX performed the experiments and drafted the manuscript. SC and YuY the acquired data, performed statistical analysis, and analyzed and interpreted the data. YgY provided follow-up information of the patients, and analyzed and interpreted the data in the revised manuscript. KS conceived and designed the research, and revised the manuscript for important intellectual content.

\section{Ethics approval and consent to participate}

The present study was approved by the Medical Ethics Committee of Xinhua Hospital (Shanghai, China). Written informed consents were provided by the participants or their legal guardians.

\section{Patient consent for publication}

Written informed consent was obtained from all patients or their legal guardians for the publication of any identifiable data or images included in this article.

\section{Competing interests}

The authors declare that they have no competing interests.

\section{References}

1. Loomes KM, Underkoffler LA, Morabito J, Gottlieb S, Piccoli DA, Spinner NB, Baldwin HS and Oakey RJ: The expression of Jagged1 in the developing mammalian heart correlates with cardiovascular disease in Alagille syndrome. Hum Mol Genet 8: 2443-2449, 1999.

2. Giannakudis J, Röpke A, Kujat A, Krajewska-Walasek M, Hughes H, Fryns JP, Bankier A, Amor D, Schlicker M and Hansmann I: Parental mosaicism of JAG1 mutations in families with Alagille syndrome. Eur J Hum Genet 9: 209-216, 2001.

3. Tada M, Itoh S, Ishii-Watabe A, Suzuki T and Kawasaki N: Functional analysis of the Notch ligand Jagged1 missense mutant proteins underlying Alagille syndrome. FEBS J 279: 2096-2107, 2012.
4. Bauer RC, Laney AO, Smith R, Gerfen J, Morrissette JJ, Woyciechowski S, Garbarini J, Loomes KM, Krantz ID, Urban Z, et al: Jagged1 (JAG1) mutations in patients with tetralogy of Fallot or pulmonic stenosis. Hum Mutat 31: 594-601, 2010.

5. Krantz ID, Piccoli DA and Spinner NB: Clinical and molecular genetics of Alagille syndrome. Curr Opin Pediatr 11: 558-564, 1999.

6. Li L, Krantz ID, Deng Y, Genin A, Banta AB, Collins CC, Qi M, Trask BJ, Kuo WL, Cochran J, et al: Alagille syndrome is caused by mutations in human Jagged1, which encodes a ligand for Notch1. Nat Genet 16: 243-251, 1997.

7. Oda T, Elkahloun AG, Pike BL, Okajima K, Krantz ID, Genin A, Piccoli DA, Meltzer PS, Spinner NB, Collins FS and Chandrasekharappa SC: Mutations in the human Jagged1 gene are responsible for Alagille syndrome. Nat Genet 16: 235-242, 1997.

8. McDaniell R, Warthen DM, Sanchez-Lara PA, Pai A, Krantz ID, Piccoli DA and Spinner NB: NOTCH2 mutations cause Alagille syndrome, a heterogeneous disorder of the notch signaling pathway. Am J Hum Genet 79: 169-173, 2006.

9. Penton AL, Leonard LD and Spinner NB: Notch signaling in human development and disease. Semin Cell Dev Biol 23: 450-457, 2012.

10. Leonard LD, Chao G, Baker A, Loomes K and Spinner NB: Clinical utility gene card for: Alagille Syndrome (ALGS). Eur J Hum Genet 22, 2014 (doi: 10.1038/ejhg.2013).

11. Turnpenny PD and Ellard S: Alagille syndrome: Pathogenesis, diagnosis and management. Eur J Hum Genet 20: 251-257, 2012.

12. Kamath BM, Bauer RC, Loomes KM, Chao G, Gerfen J, Hutchinson A, Hardikar W, Hirschfield G, Jara P, Krantz ID, et al: NOTCH2 mutations in Alagille syndrome. J Med Genet 49: 138-144, 2012.

13. Warthen DM, Moore EC, Kamath BM, Morrissette JJ, Sanchez-Lara PA, Piccoli DA, Krantz ID and Spinner NB: Jagged1 (JAG1) mutations in Alagille syndrome: Increasing the mutation detection rate. Hum Mutat 27: 436-443, 2006

14. Boyer J, Crosnier C, Driancourt C, Raynaud N, Gonzales M, Hadchouel $\mathrm{M}$ and Meunier-Rotival M: Expression of mutant JAGGED1 alleles in patients with Alagille syndrome. Hum Genet 116: 445-453, 2005.

15. Boyer-Di Ponio J, Wright-Crosnier C, Groyer-Picard MT, Driancourt C, Beau I, Hadchouel M and Meunier-Rotival M: Biological function of mutant forms of JAGGED1 proteins in Alagille syndrome: Inhibitory effect on Notch signaling. Hum Mol Genet 16: 2683-2692, 2007.

16. Morrissette JD, Colliton RP and Spinner NB: Defective intracellular transport and processing of JAG1 missense mutations in Alagille syndrome. Hum Mol Genet 10: 405-413, 2001.

17. Liu C, Yang C, Lu L, Wang W, Tan W, Leung CH and Ma DL: Luminescent iridium(iii) complexes as COX-2-specific imaging agents in cancer cells. Chem Commun (Camb) 53: 2822-2825, 2017.

18. Lincoln R, Greene LE, Zhang W, Louisia S and Cosa G: Mitochondria alkylation and cellular trafficking mapped with a lipophilic BODIPY-acrolein fluorogenic probe. J Am Chem Soc 139: 16273-16281, 2017.

19. Lin S, Gao W, Tian Z, Yang C, Lu L, Mergny JL, Leung CH and Ma DL: Luminescence switch-on detection of protein tyrosine kinase-7 using a G-quadruplex-selective probe. Chem Sci 6: 4284-4290, 2015

20. Lu F, Morrissette JJ and Spinner NB: Conditional JAG1 mutation shows the developing heart is more sensitive than developing liver to JAG1 dosage. Am J Hum Genet 72: 1065-1070, 2003.

21. Zhang E, Hong N, Chen S, Fu Q, Li F, Yu Y and Sun K: Targeted sequencing identifies novel GATA6 variants in a large cohort of patients with conotruncal heart defects. Gene 641: 341-348, 2018.

22. Pu T, Liu Y, Xu R, Li F, Chen S and Sun K: Identification of ZFPM2 mutations in sporadic conotruncal heart defect patients. Mol Genet Genomics 293: 217-223, 2018.

23. Livak KJ and Schmittgen TD: Analysis of relative gene expression data using real-time quantitative PCR and the 2(-Delta Delta C(T)) method. Methods 25: 402-408, 2001.

24. Li L, Dong J, Wang X, Guo H, Wang H, Zhao J, Qiu Y, Abuduxikuer K and Wang J: JAG1 mutation spectrum and origin in chinese children with clinical features of alagille syndrome. PLoS One 10: e0130355, 2015.

25. Grochowski CM, Loomes KM and Spinner NB: Jagged1 (JAG1): Structure, expression, and disease associations. Gene 576: 381-384, 2016 
26. Chillakuri CR, Sheppard D, Lea SM and Handford PA: Notch receptor-ligand binding and activation: Insights from molecular studies. Semin Cell Dev Biol 23: 421-428, 2012.

27. Cordle J, Johnson S, Tay JZ, Roversi P, Wilkin MB, de Madrid BH, Shimizu H, Jensen S, Whiteman P, Jin B, et al: A conserved face of the Jagged/Serrate DSL domain is involved in Notch trans-activation and cis-inhibition. Nat Struct Mol Biol 15: 849-857, 2008.

28. Lykke-Andersen S and Jensen TH: Nonsense-mediated mRNA decay: An intricate machinery that shapes transcriptomes. Nat Rev Mol Cell Biol 16: 665-677, 2015.

29. He F and Jacobson A: Nonsense-mediated mRNA decay: Degradation of defective transcripts is only part of the story. Annu Rev Genet 49: 339-366, 2015.

30. Mocquet V, Durand S and Jalinot P: How retroviruses escape the nonsense-mediated mRNA decay. AIDS Res Hum Retroviruses 31: 948-958, 2015.

31. Neu-Yilik G, Amthor B, Gehring NH, Bahri S, Paidassi H, Hentze MW and Kulozik AE: Mechanism of escape from nonsense-mediated mRNA decay of human beta-globin transcripts with nonsense mutations in the first exon. RNA 17: 843-854, 2011

32. Ferraiuolo MA, Lee CS, Ler LW, Hsu JL, Costa-Mattioli M, Luo MJ, Reed R and Sonenberg N: A nuclear translation-like factor eIF4AIII is recruited to the mRNA during splicing and functions in nonsense-mediated decay. Proc Natl Acad Sci USA 101: 4118-4123, 2004.
33. Neu-Yilik G, Gehring NH, Thermann R, Frede U, Hentze MW and Kulozik AE: Splicing and 3'end formation in the definition of nonsense-mediated decay-competent human beta-globin mRNPs. EMBO J 20: 532-540, 2001.

34. Yuan ZR, Kobayashi N and Kohsaka T: Human Jagged 1 mutants cause liver defect in Alagille syndrome by overexpression of hepatocyte growth factor. J Mol Biol 356: 559-568, 2006.

35. Sparks EE, Huppert KA, Brown MA, Washington MK and Huppert SS: Notch signaling regulates formation of the three-dimensional architecture of intrahepatic bile ducts in mice. Hepatology 51: 1391-1400, 2010.

36. High FA and Epstein JA: The multifaceted role of Notch in cardiac development and disease. Nat Rev Genet 9: 49-61, 2008.

37. Humphreys R, Zheng W, Prince LS, Qu X, Brown C, Loomes K, Huppert SS, Baldwin S and Goudy S: Cranial neural crest ablation of Jagged1 recapitulates the craniofacial phenotype of Alagille syndrome patients. Hum Mol Genet 21: 1374-1383, 2012.

38. Kamath BM, Bason L, Piccoli DA, Krantz ID and Spinner NB: Consequences of JAG1 mutations. J Med Genet 40: 891-895, 2003. 